\title{
Repensar digitalmente un museo desde la sostenibilidad; la experiencia "Vi är planeten"
}
Digitally rethink a museum from sustainability; the "Vi är planeten" experience

\author{
José Antonio Gordillo Martorell \\ Museipedagog-jose.gordillo-martorell@norrbotten.se
}

\begin{abstract}
Resumen
La exposición "Vi är planeten" (Somos planeta) inaugurada el 24 de noviembre de 2020 en el Norrbottens Museum fue concebida inicialmente como una exposición sobre sostenibilidad basada en la experiencia de la interacción física del público. Sin embargo, el impacto del Corona y el cierre temporal del Museo obligó a repensar la manera en la que la exposición debía comunicarse con el público desde la experiencia digital. El reto consistía en alcanzar los objetivos de engagement, aprendizaje, motivación y cambio de percepción en torno a la sostenibilidad que el equipo educativo se había planteado alcanzar inicialmente a través de la visita fisica del público empleando una estrategia digital eficiente.

Uno de los campos en los que hemos planteado una nueva forma de crear una experiencia digital completa significativa ha sido el de las visitas digitales de grupos escolares a la exposición. Esta comunicación presenta el modelo de visita que hemos creado para los estudiantes suecos de Primaria, Secundaria y Bachillerato, los retos a los nos hemos enfrentado, asi como algunos de los resultados provisionales que estamos obteniendo. Finalmente, apuntamos algunas conclusionesw provisionales que pueden resultarles útiles a roifesionales que estén trabajndo con estos formatos.
\end{abstract}

Palabras clave: Sostenibilidad, co-creación, aprendizaje, digital, evaluación.

\footnotetext{
Abstract

The exhibition "Vi är planeten" (We are planet) inaugurated on November 24, 2020 at the Norrbottens Museum was initially conceived as an exhibition on sustainability based on the experience of the physical interaction of the public. However, the impact of the Corona and the temporary closure of the Museum forced us to rethink the way in which the exhibition should communicate with the public from the digital experience. The challenge consisted in achieving
} 
Repensar digitalmente un museo desde la sostenibilidad; la experiencia "Vi är planeten"

the objectives of engagement, learning, motivation and change of perception around sustainability that the educational team had initially set out to achieve through the physical visit of the public using an efficient digital strategy.

One of the fields in which we have come up with a new way of creating a meaningful full digital experience has been the digital visits of school groups to the exhibition. This communication presents the visiting model that we have created for Swedish Primary, Secondary and Baccalaureate students, the challenges we have faced, as well as some of the provisional results that we are obtaining. Finally, we note some provisional conclusionsw that may be useful to professionals who are working with these formats.

Keywords: Sustainability, co-creation, learning, digital, evaluation. 


\section{Introducción}

El 25 de noviembre de 2020 se inauguraba la exposición "Vi är planeten" (Somos planeta" en el Norrbottens Museum (Norrbottens Museum, 2020). Se trata de un proyecto dirigido a cambiar la percepción que el público tienen sobre la sostenibilidad en dos sentidos: poniendo en cuestión el discurso "apocalipticista" que circula en medios de comunicación y redes sociales en torno a temas relacionados con la sostenibilidad como el Cambio Climático ("Ya es demasiado tarde para poder hacer algo etc...”), y aproximando este tema a la vida cotidiana de las personas conectando los Objetivos Globales de Sostenibilidad con su forma de comer, comprar, consumir, trabajar, educarse o colaborar. Seguíamos en ese sentido el planteamiento de Nina Simon de generar experiencias relevantes en nuestros visitantes (Simon, 2016).

La exposiciónn se concibió desde el principio como un espacio híbrido -parte espacio expositivo, parte Hub, parte Laboratorio/Incubadora- en el que lo importante era lo que el visitante podía hacer a partir de ese cambio de percepción que planteábamos generada por la experiencia significativa que iba a vivir in situ. El reto era doble. Por un lado queríamos que cada visitante encontrara algo que conectara con algo significativo para su vida cotidiana. Esta conexión era el primer paso y se planteaba como principal resultado a obtener tras la visita a la exposición. A partir de ahí queríamos establecer un vínculo emocional del visitante con la exposición invitándolo a co-crear el påropio espacio expositivo aportando sus ideas, generando nuevos proyectos y/o debatiendo. Nuestro ideal era que la exposición estuviera constantemente co-creándose con las aportaciones ofrecidas por parte de cada uno de los visitantes. Aspirábamos a tener cada día una exposición diferente. Queríamos tantas exposiciones como visitantes tuviera la exposición conformando una especie de organismo vivo que evolucionara y se transformara a lo largo de sus dos años de vida.
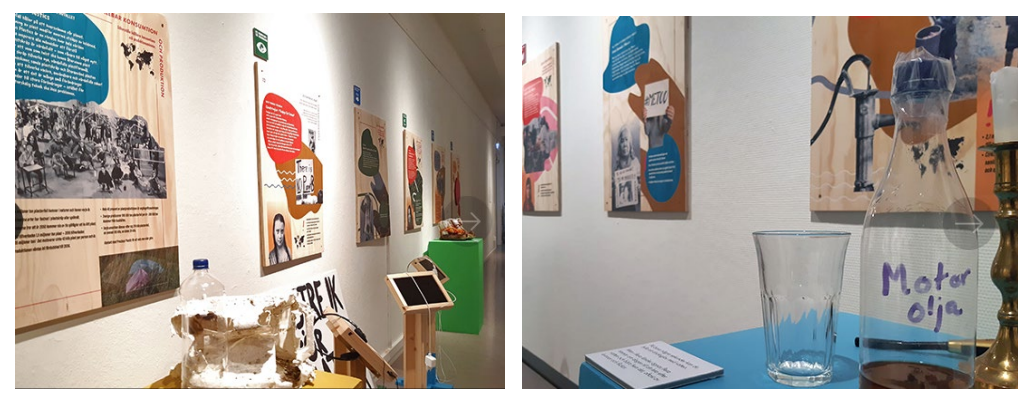

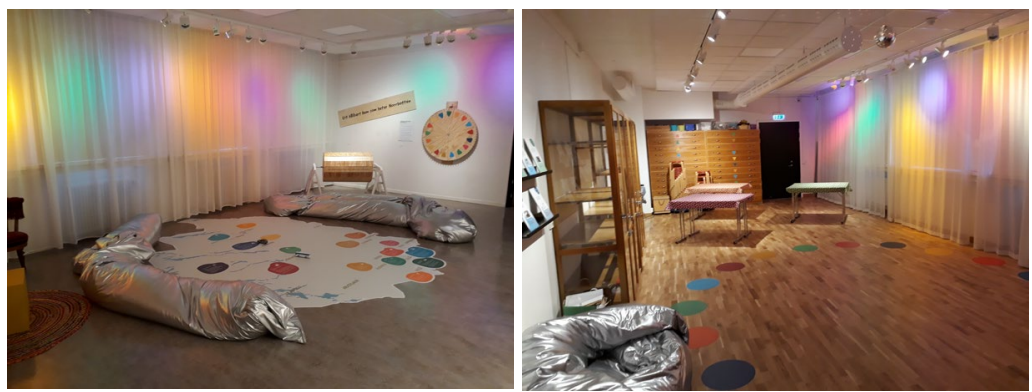

Fig.1 Distintas zonas de la exposición "Vi är planeten" Fuente: Norrbottens Museum (2020)

Para hacer frente al primer reto generamos una serie de historias inspiradoras de personas provinientes de distintas partes del mundo -una para cada Objetivo Global de Sostenibilidadque habian conseguido hacer algo significativo en el terreno de la sostenibidilidad. También creamos dinámicas de gamificación empleando ruletas, tómbolas, grandes volúmenes de colores etc.. con las que garantizar un engagement para algunos de nuestros públicos objetivos (niños y jóvenes). Para el segundo reto combinamos metodologias basadas en el Do It Yourself, el Design Thinking en un espacio llamado FabLab enfocado hacia el prototipado de ideas, y la dinámica de debates públicos y la confrontación de argumentos bien informados sobre problemas globales con manifestación local, en una zona en la que creamos una réplica de la Asamblea General de las Naciones Unidas.

Estos principios han sido básicos a la hora de hacer una transición rápida y eficiente hacia el contexto digital desde el que nos vimos forzados a trabajr en exclusiva desde que la pandemia hizo su aparición. Sin su guía dicha transición no hubiera sido posible. Queremos subrayar este punto. Sin un armazon teórico, conceptual y estratégico previos que nos indicaba el por qué y el qué no hubiésemos conseguido llegar al cómo que fue la parte en la que intervienieron de forma complementaria y a posteriori las herramientas digitales de las que nos dotamos para llevar a cabo las viistas a la exposición.

\section{Objetivos}

Los objetivos pricipales que nos marcamos desde el principio con "Vi är planeten" fueron de tres tipos:

Objetivos relacionados con la percepción del público en torno a la sostenibilidad:

- Transformar la percepción negativa y excesivamente oficial sobre sostenibilidad en algo relevante, interesante y apasionante para cada uno de los visitantes.

- Generar oportunidades de creación y aprendizaje que permitan adquirir comptencias y habilidades relacionadas con la sostenibilidad como la creatividad cooperativa, el pensamiento crítico a largo plazo, los enfoques holísticos o el networking. 
Objetivos relacionados con el vínculo del público con la exposición:

- Reforzar el componente emocional del vínculo a través de estimulos sensoriales como la música o la imagen.

- Aportar innovaciones en el engagement digital de los visitantes a la exposición empleando herramientas como los mapas digitales, las Apps educativas, los videojuegos o el diseño y la impresión digital.

- Objetivos relacionados con la implicación del público con su comunidad local a partir de la experiencia de visita de la exposición:

- Transformar a la región que alberga la exposición -Norrbotten- en una gran laboratorio-aula en la que experimentar de formar individual o colectiva qué es la sostenibilidad, por qué es importante y cómo podemos contribuir cada uno de nosotros a ella haciendo algo con otras personas de la región.

Cada uno de los objetivos iba asociado a una serie de indicadores cuantitativos y cualitativos con los que poder medir el progreso en los mismos y llevar a cabo medidas de corrección en el caso de que éstas fueran necesarias.

El público escolar contaba con su propia serie de indicadores y su propia metodología de evaluación. El resultado principal que buscábamos en los alumnos era por un lado un cambio de percepción de la sotenibilidad que les llevara a un cambio de comportamiento en su vida diaria. Y por otro, iniciar un proceso creativo con el que podían contribuir a través de sus ideas e iniciativas a un futuro mäas sostenible para todos. De estas dos formas se atacaban las dos inercias relacionadas con la sostenibilidad en las que el estudiante suelen verse envueltos a partir de los inputs que reciben constantemente desde su entorno, televisión e internet etc...: entender la sostenibilidad en clave catastrofista y considerarla como algo negativo que va siempre asociado a la palabra "problema grave".

\section{Desarrollo de la innovación}

Desde diciembre de 2020 llevamos a cabo nuestras primieras visitas digitales a la exposición dirigidas a grupos escolares. Empleamos Teams como programa de referencia dado que la mayoría de los centros trabajan con este programa. Fijamos una hora de duración de la visita y la planteamos de una forma muy flexible adaptándonos a las necesidades educativas que cada profersor manifestaba en torno al tema de la sostenibilidad y a la manera en la que lo trabajaba en el aula. La visita la planteábamos como una especie de "gran bufé" en el que cada profesor podía elegir que Objetivos Globales de Sostenibilidad quería trabajar más en concreto y de qué manera. Para ello nos fue de especial ayuda la espiral de la inadagación de Linda Kaser y Judy Halbert (Kaser et Halbert, 2017) que empleamos como metodología de trabajo de referencia siguiendo algunas de sus fases principales.

Comenzamos con grupos de Primaria con una visita digital dramatizada guiaba por un astronauta de la Estación Espacial Internacional que había venido al Museo con un mensajem uy importante para los alumnos: somos planeta y no debenmos olvidarlo. Desde el espaco no 
se ven fronteras, s'lo un mundo azul hernoso y único. Lo que le hagamos al planeta, nos lo hacemos a nosotros mismos y a la inversa. En estas visitas nos centramos en los OGS 7 y 13 trabajando con historias apasionantes como la de la inventora Jessica Lin y su balón de futbol capaz de producir electricidad con la que niños surafricanos podían estudiar sus lecciones después de clase. Explicamos conceptos básicos como el calentamiento global o el efecto invernadero empleando objetos cotidianos como el aceite de un coche y realizamos dinámicas de brain storming para crear juntos "listas de compromiso con la sostenibilidad" donde nos marcábamos una serie de retos como comer menos carne, tirar menos comida, ir en bicicleta al colegio, apagar las luces cuando no fueran necesarias etc... que revisaríamos al mes de la visita. Esta lista se complementaba con otra sobre formas de generar electricidad de una forma sostenible en la que los alumnos daban rienda suelta a su creatividad planteando desde gadgets acoplados al movimiento de su cuerpo hasta sistemas para aprovechar el movimiento de un palo de hockey en un partido. Todo empezaba a ser posible.

En febrero de 2021 basándonos en la experiencia previa de estos niveles iniciamos el diseño de las visitas guiadas para Bachillerato (Gymnasiet). Introdujimos varias modificaciones importantes para hacer la experiencia de visita digital más eficiente:

- Necesitábamos emplear recursos multimedia que podríamos "importar" de la propia exposición para hacerlos más atractivos y sobre todo para conectar con las emociones de los alumnos desde el principio. Por ejemplo decidimos iniciar la visita preguntándoles cuál es el sonido del Cambio Climático para a continuación pedirles que cerraran los ojos y trataran de adivinar qué era lo qaue estaban escuchando que no era otra cosa que el estruendo de un glaciar derritiéndose en la Antártida.

- Los alumnos debían implicarse en la visita de forma activa haciendo cosas, no sólo recibiendo información de forma pasiva (del mismo modo que lo planteábamos para el caso de la visita física). Muchos de ellos padecían la famosa "fatiga de Zoom" (Bailenson, 2021) por lo que era necesario plantear la interacción digital de una forma muy dinámica donde desde el principio ellos hicieran cosas: debatir, analizar, investigar, buscar información, representar, hacer de portavoces, exponer, comentar etc... Para ello y además del diseño de las dinámicas nos resultó de mucha ayuda herramientas colaborativas como Miro o Mural como facilitadoras de la co-creación de ideas.

- Otro aspecto importante relacionado con la fatiga de Zoom era la fatiga emocional de los alumnos provocada por la experiencia dura del Covid. Las visitas digitales eran una forma de lanzar a los alumnos un mensaje de atención y cuidado. Haciendo estas visitas veníamos a transmitirles que nos importaban, que podíamos hacer algo interesante juntos y que incluso podrían salir reforzados emocionalmente de la experiencia que estaban atravesando. Como bien describe la profesora del MIT Canan Dagdeviren a la hora de diseñar nuestra estrategia digital era fundamental tener presente el factor humano de nuestros alumnos no contribuyendo a estresarlos con una lección mäas en formato digital sino todo lo contrario, aliviando en la medida de lo posible su ansiedad recurriendo a la empatía (Dagdeviren, 2020). 
- La sostenibilidad como nuevo contenido currricular que tratábamos de conectar con el programa clásico implicaba entrenar nuevas competencias: desde Design Thinking a habilidades comunicativas relacionadas con el Storytelling, desde el pensamiento crítico a la creación de equipos, desde la visión holística al uso de herramientas digitales. Nos planteamos la visita como la formaen la que los alumnos comenzarían a familiarizarse con esta aptitudes utilizándolas en las dinámicas que preparamos para ellos a lo largo de la visita de manera que pudieran continuar trabajando con ellas a la hora de llevar a cabo los retos que les lanzamos a un mes vista.

- Uns aspecto clave en el enfoque de la visita fue entenderla como una parte de un proceso de aprendizaje más amplio que podía llegar a extenderse hasta un mes después del primer encuentro digital. La secuencia se iniciaba con una entrevista personalizada con el profesor a quien se le explicaba bien toda la secuencia y el por qué de la misma junto con los recursos a los que iba a tener acceso digitalmente. Se procedía a la visita. Se daba un margen de un mes para que los alumnos llevaran a cabo los desafíos que se les plantea y finalmente se llevaba a cabo una segunda reunión digital protagonizada completamente por los alumnos en la que presentarían sus propuestas, ideas y proyectos. En sí esta segunda reunión es una reunión completamente evaluativa para nosotros pero también para el profesor y para los alumnos. Es el momento de demostrar y demostrarse a sí mismo lo que han aprendido. Se trata pués de una línea de trabajo muy ajustada, hecha a medida de las necesidades de los alumnos y del profesor, en la que en un momento determinado (cuando el alumno vuelve al entorno físico) el alumno toma el control de su propio proceso de aprendizaje.

- Por último, aprendimos que digital no significa exclusividad mas bien al contrario: lo digital puede entenderse como un camino de ida y vuelta interesante con el mundo físico por el que el alumno puede deambular con provecho. El alumno parte de una serie de estímulos que le ofrecemos en nuestro primer encuentro digital para a partir de ahí enfrentarse al mundo físico desde el que llevará a cabo su proyecto. Después retornará al entorno digital para "presentar dicho proyecto en sociedad". El proyecto lo puede hacer de forma individual o colectiva y puede completarlo posteriomente viniendo a la zona del FabLab del Museo empleando herramientas como App de diseño digital o impresoras 3D siguiendo el Codesign generative Cycle de Liz Sanders (SANDERS et STAPPERS, 2013).

\section{Resultados}

Presentamos dos ejemplos concretos de resultados correspondientes a dos visitas digitales digitales ofrecidas a alumnos de Bachillerato con dos especialidades distintas: lengua e idiomas y Hotel y turismo.

En el caos de los estudiantes de lengua e idiomas el proyecto en el que trabajaron durante el mes posterior a la vista vinculado al SDG 13 fue el diseño de una solución tecnológica 
dirigida a paliar el efecto local del fenómeno de retroceso y deshielo del gaclar Kebnekaise en el norte de Suecia. ¿Còmo podemos utilizar la tecnología para revertir de algún modo el impacto del calentamiento global en todo el norte de Europa y concretamente en la zona en la que viven los estudiantes?

Durante la visita se les presentó como ejemplo inspirador el proyecto Ice Stupa (SECMOL School, 2019) que afrontaba un reto similar en la zona del Himalaya, concretamente en la región de Ladakh. Ice Stupa se centra en la creación de glaciares artificiales para congelar y retener el agua que sigue fluyendo y consumiéndose por los arroyos y los ríos durante el invierno. Es un buen ejemplo de cooperación entre científicos, ingenieros y la comunidad local para hacer frente con ciencia y tecnología a uno de los mayores desafíos del impacto del Cambio Climático en la zona y en toda India: la falta de agua y la supervivencia de los campesinos locales.

Por su parte estudiantes de Turismo trabajaron sobre otra area de contenido relacionada con los SDG 8 y 12 centrándose en el concepto de comercio justo. El proyecto que se planteó para presentar al mes de la visita fue el diseño de una tienda virtual en la que se ofrecerían seis productos (dos relacionados con la alimentación, dos con prendas de ropa y el movimiento "slow fashion "y dos productos de artesanía local) obtenidos a través de las redes de comercio justo y que debían cumplir con los estandares de condiciones laborares decentes para sus productores y de impacto medioambiental respecto de los materiales para crearlos.

Una parte fundamental que debían trabajar los alumnos era la de la información proporcionada al cliente de la tienda. Durante la visita digital una idea central sobre la que se trabajó de manera explícita fue la del poder del cosumidor bien informado. Uno de los objetivos de la visita era facilitarle al alumno otros criterios que le permitieran tomar una decisión más formada y profunda a la hora de realizar sus compras considerando además del factor precio o los gustos estéticos personales, otros factores como las condiciones laborales de las trabajadoras o el impacto mediomabiental de los productos en venta. Era imporante que en su proyecto pensaran como iban a informar sobre estos aspectos al potencial comprador. El proyecto iba además a tener continuidad en el tiempo planteándose includo la posibilidad de plantearlo como el embrión de una futura start up.

\section{Conclusiones}

Le experiencia de las visitas digitales que estamos llevando a cabo hasta la fecha apunta una serie de conclusiones provisionales que nos gustaría compartir con profesionales que llevan a cabo en la actualidad esta línea de trabajo en sus respectivas organizaciones:

- El soporte digital en sí no puede suplir un marco teórico y metodológico previo que guíe la visita sobre todo en lo que respecta a cómo entendemos el aprendizaje. Sin ese armazón la experiencia no tendrá la calidad que se requiere por muy buenos recursos técnicos que se disponga. 
- Es fundamental conocer lo mejor posible antes de la visita al público con el que vamos a interaccionar e integrar ese conocimiento en nuestra estrategia de visita y de mediación.

- Debemos estar abiertos a los inputs que recibimos de nuestro público digital a todos los niveles para reajustar nuestra visita; desde aspectos de tipo práctico como la duración, el sonido o la iluminación hasta cuestiones más de fondo como los contenidos en los que nos queremos centrar, los conceptos que queremos presentar o los recursos de la propia exposición que queremos visibilizar. La demostración de que estamos haciendo bien nuestro trabajo es que iremos contando rápidamente con versiones mejoradas de la visita con las que poder trbajar. Versiones que van incorporando aquello que funciona mejor y corrigen lo que funciona peor.

- Tener en consideración aspectos psicológicos del visitante (especialmente con alumnos) es fundamental para que la visita funcione. Vamos a enfrentarnos (esto es especialmente cierto en el caso de estudiantes) a públicos con situaciones personales muy complicadas que posiblemente atraviesen por momentos dificiles. Debemos aportar un extra de empatía en nuestras visitas que lance el mensaje de que somos sensibles a la situación individual por la que está atravesando cada uno de nuestros visitantes.

- La visita digital a la exposición exige contar con algunos de los recursos físicos de la exposición transformándolos en clave digital. Hay que evitar el riesgo de "olvidar" la exposición física en favor de la versatilidad digital. La exposición física debe ser en última instancia un marco de referencia sobre el que poder trabajar problemáticamente con el público, no de manera mecánica.

- Lo digital debe combinarse con lo físico de una forma equilibrada. Lo digital puede ser un buen punto de partida desde el que acudir a lo físico para llevar a cabo proyectos con materiales y recursos tangibles, para desde ahí volver de nuevo a lo digital como plataforma en la que poder presentar, comunicar, debatir e incluso mejorar la versión del proyecto realizada físicamente. No debemos olvidar la necesidad de experiencia material de nuestros visitantes, alqo que nos reclaman y que no podemos hurtarles amparándonos en una supuesta exclusividad digital. 


\section{Referencias}

BAILENSON, J. N. (2021) "Nonverbal Overload: A Theoretical Argument for the Causes of Zoom Fatigue" en Technology, Mind and Behaviour, Vol. 2, Issue 1.

DAGDEVIREN, C. (2020) "Remote Teaching and Learning at the Media Lab" en Medium, $<$ https://medium.com/mit-media-lab/remote-teaching-and-learning-at-the-media-lab706fc830e406> [Consulta: 16 de diciembre de 2020].

KASER, L. y HALBERT, J. (2017). The Spiral Playbook: Leading with an inquiring mindset in school systems and schools. Canada. C21.

NORRBOTTENS MUSEUM. Vi är planeten

$<$ https://norrbottensmuseum.se/evenemang/utstaellningar/vi-aerplaneten.aspx?lang=en> [Consulta: 25 de noviembre de 2020].

SANDERS, L. y STAPPERS, P.J. (2013) Convivial Toolbox: Generative Research for the Front End of Design. London. Laurence King Publishing.

SECMOL SCHOOL. The Ice Stupa Project $<$ http://icestupa.org/> [Consulta: 16 de junio de 2019].

SIMON, N. (2016). The Art of Relevance. Santa Cruz, Califorina. Museum 2.0. 